\title{
Performance of HARQ with Reduced Size Retransmissions using Network Coding Principles
}

\author{
Matthias Woltering, Dirk Wübben and Armin Dekorsy \\ University of Bremen, Bremen, Germany \\ Email: \{woltering, wuebben, dekorsy\}@ant.uni-bremen.de
}

\author{
Volker Braun and Uwe Doetsch \\ Alcatel-Lucent Bell Labs, Stuttgart, Germany \\ Email: \{volker.braun, uwe.doetsch\}@alcatel-lucent.com
}

\begin{abstract}
This paper discusses retransmission approaches to improve the throughput performance of Hybrid-ARQ (HARQ) schemes in a point-to-point single user 3rd Generation Partnership Project (3GPP) Long Term Evolution (LTE) uplink system. One goal of communication systems is to achieve a reliable transmission with a throughput performance as close as possible to channel capacity. For that, reducing the channel utilization will improve the throughput performance. Instead of sending two retransmission packets for two HARQ processes of one users, a previously published HARQ scheme uses the XOR combining of these packets to get only one retransmission packet with the same size. Similar to this idea, a new varied scheme performs XOR combining of parts of one conventional full retransmission to generate a smaller retransmission packet. Both approaches will reduce the channel utilization. They will be compared with a HARQ system in LTE uplink using a full size retransmission and a half size retransmission. The main focus of this work is the throughput performance evaluation of these schemes in an LTE link-level simulator.
\end{abstract}

Index Terms-Network coding, HARQ, In-Packet, LTE uplink.

\section{INTRODUCTION}

Throughput performance is an important value in communication systems. It is the average rate of successful transmissions of information. The theoretical bound of throughput is the channel capacity introduced by Shannon. One goal of communication systems is a reliable transmission with throughput as close as possible to capacity. For the reliable transmission HARQ protocols are introduced. In case of a decoding failure the transmitter sends a retransmission, which should help in the decoding process. The standard of 3GPP LTE allows a variable use of retransmission packet size to achieve a better throughput, but the adaptive use leads to a higher impact on control signaling overhead. For that, we only look on a fixed reduction of retransmission size by a factor of two. This will be compared with a HARQ system using always a full size retransmission, here called HARQ full ReTx.

Within this paper we present two approaches of reducing the size of Hybrid-ARQ retransmission packets simulated in LTE uplink [1], but they are not confined to LTE or uplink. The proposed schemes reduce either the retransmission size by using XOR combination within one codeword, which is motivated by network coding (NC) or by implementing an additional puncturing.

In a multicast scenario, $\mathrm{NC}$ has been proposed as a scheme to combine several packets as one packet [2]. For wireless communication with intermediate nodes it has been shown that combining of packets of two nodes could also be useful to improve the throughput performance [3]. The combination of HARQ and NC has been studied for multicast multiuser scenarios with relays in [4] and [5]. There, the authors have applied the NC principle across packets of different users. Corresponding throughput improvements by using this NCHARQ principle has been demonstrated in [6] and [7]. In contrast, another approach to use NC principle was published in [8] and [9]. Herein, the idea is to use the XOR combination, similar to NC, of two packets of different HARQ processes into one retransmission. For that, a retransmission packet in NC-HARQ has the same size then one packet in a HARQ full ReTx, but it contains a combination of information of two different messages. NC-HARQ has shown benefits vs. HARQ full ReTx, but was not yet compared to HARQ half ReTx.

In this paper, we introduce a varied version of the previous published scheme [8] and [9]. Instead of sending a XOR combined retransmission packet with the same size as HARQ full ReTX, we transmit a retransmission packet, formed by a XOR combination of parts of one retransmission packet generated by the common structure in LTE, called NC-InPacket. This approach reduces the size of the retransmission and therefore improves the throughput performance. We also introduce a further reduced size retransmission scheme as benchmark, called HARQ half ReTx. Here the user equipment (UE) creates a retransmission packet by using an additional puncturing on top of the HARQ full ReTx packet.

The remainder of this paper is organized as follows. Section II introduces the general system model for uplink transmission. In Section III both proposed retransmission schemes are introduced. The iterative decoding algorithm for NC-InPacket is presented in Section IV. The throughput performance is investigated by means of simulation results in Section V. Conclusions follow in Section VI.

\section{SYSTEM MODEL IN LTE UPLINK}

\section{A. Mathematical Description}

In this paper, we consider XOR combination strategies similar to NC in order to reduce the retransmission size in a LTE point-to-point uplink scenario [1]. An UE transmits a packet $\mathbf{c}_{i}$, with $i$ as codeword index to an evolved Node B (eNB) which is generated by an LTE turbo encoder [1], as shown in Fig. 1. The encoder structure converts the binary information vector $\mathbf{a}_{i} \in \mathbb{F}_{2}^{N_{a}}$ of length $N_{\mathrm{a}}$ to three streams: a 
systematic part and two parity parts. These streams are individually interleaved and fed into a circular buffer. The generated mother code $\mathbf{b}_{i}$ with length $N_{\mathrm{b}}=3 \cdot N_{\mathrm{a}}$ has the mother code rate $R=N_{\mathrm{a}} / N_{\mathrm{b}}=1 / 3$. Out of this mother codeword, the rate matching block selects a number of bits depending on the redundancy version (RV) index $r$ and constructs the codeword $\mathbf{c}_{i}^{r} \in \mathbb{F}_{2}^{N_{\mathrm{c}}}$ of the transmitted packet with length $N_{\text {c }}$. Two different retransmission philosophies are possible, Chase Combining (CC) has always the same RV index $r$ and Incremental Redundancy (IR) changes the RV index $r$ for every retransmission, i.e, with every new retransmission IR selects other bits as the previous retransmission. Hence, the effective code rate for both philosophies is given by $R_{\mathrm{C}}^{r}=\frac{N_{\mathrm{a}}}{N_{\mathrm{c}}}$.

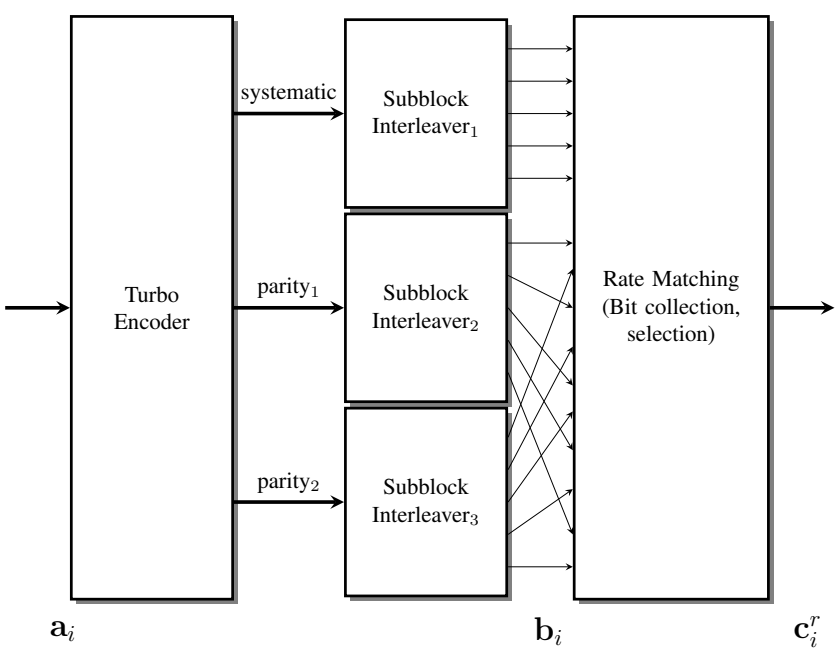

Fig. 1. Generation of transmit packets, after turbo encoding and interleaving the generated code bits are stored in a circular buffer. Out of this buffer the bit collection block collects bits depending on $r$, which will be sent [1].

Due to the limitation of the internal interleaver length in the LTE turbo encoder, transport formats (TFs) of length larger than 6144 bits are split into segments, i.e., the information bit vector $\mathbf{a}_{i}$ is separated into segments $\mathbf{a}_{i, s}$ with $s=1, \ldots, N_{\mathrm{s}}$ and length $N_{\mathrm{a}} / N_{\mathrm{s}}$. The number of segments $N_{\mathrm{s}}$ is determined by the TF. The structure shown in Fig. 1, is used for every segment $\mathbf{a}_{i, s}$ separately, i.e., every information part $\mathbf{a}_{i, s}$ is encoded to its codeword $\mathbf{c}_{i, s}^{r}$, separately. An additional concatenation block stacks the codewords $\mathbf{c}_{i, s}^{r}$ to the overall codeword $\mathbf{c}_{i}^{r}$ :

$$
\mathbf{c}_{i}^{r}=\left[\mathbf{c}_{i, 1}^{r}, \mathbf{c}_{i, 2}^{r}, \ldots, \mathbf{c}_{i, N_{\mathrm{s}}}^{r}\right] \in \mathbb{F}_{2}^{N_{\mathrm{c}}} .
$$

The code bits of the whole codeword $c_{i, \ell}$ with $\ell=1, \ldots, N_{\mathrm{c}}$ are mapped to symbols $x_{i, \kappa}$ of a modulation alphabet $\mathbb{X}$ (e.g., QPSK, 16-QAM or 64-QAM) and transmitted to eNB. In case of a block flat fading channel the received signal vector in time slot $t_{i}$ is given by

$$
\mathbf{y}_{i}=h_{i} \mathbf{x}_{i}+\mathbf{n}_{i}
$$

where $\mathbf{n}_{i}$ is a noise vector with $\mathbf{n}_{i} \sim \mathcal{N}\left(0, \sigma_{n}^{2}\right)$. The coefficient $h_{i}$ denotes a complex-valued zero-mean circular symmetric

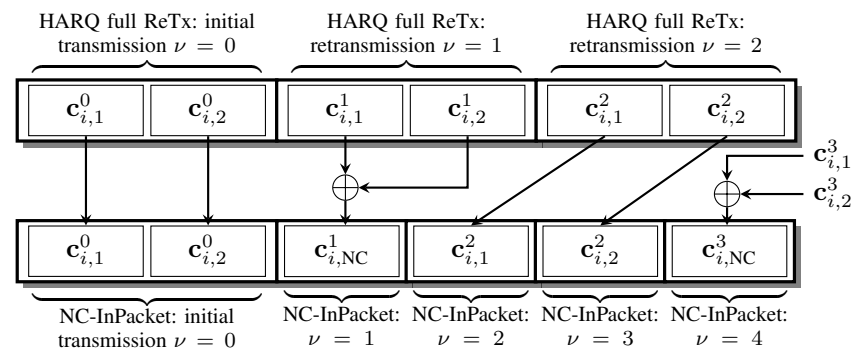

Fig. 2. Generate retransmission packet: XOR combination formed by parts $\mathbf{c}_{i, 1}^{r}$ and $\mathbf{c}_{i, 2}^{r}$ of the codeword $\mathbf{c}_{i}^{r}$, the HARQ full ReTx scheme is presented at the top of the figure with 2 retransmissions, NC-InPacket at the bottom with four retransmission. Here as example for IR. $\nu$ is the transmission index.

Gaussian distributed variable with variance one. Note that, for simulation we use more general channels.

Based on the received symbol $y_{i, \kappa}$ and the channel coefficient $h_{i}$, we calculate the log-likelihood ratio (LLR) $\mathrm{L}_{\text {Dem }}\left(c_{i, \ell}\right)$ of the participating coded bit $c_{i, \ell}$ by:

$$
\begin{aligned}
\mathrm{L}_{\text {Dem }}\left(c_{i, \ell}\right) & =\ln \frac{\operatorname{Pr}\left\{c_{i, \ell}=0 \mid y_{i, \kappa}, h_{i}\right\}}{\operatorname{Pr}\left\{c_{i, \ell}=1 \mid y_{i, \kappa}, h_{i}\right\}} \\
& =\ln \frac{\sum_{x \in \mathbb{X}, c_{i, \ell}=0} \exp \left(\frac{-\left|y_{i, \kappa}-h_{i} x\right|^{2}}{\sigma_{n}^{2}}\right)}{\sum_{x \in \mathbb{X}, c_{i, \ell}=1} \exp \left(\frac{-\left|y_{i, \kappa}-h_{i} x\right|^{2}}{\sigma_{n}^{2}}\right)} .
\end{aligned}
$$

\section{RETRANSMISSION SCHEMES}

This section describes the encoder for the schemes with reduced size retransmission (NC-InPacket and HARQ half ReTx). The impact of system parameters of both schemes will be explained in comparison to the NC-HARQ scheme in [8], [9] and HARQ full ReTx in [1].

\section{A. Network Coding within a Packet (NC-InPacket)}

Fig. 2 illustrates the retransmission principle used in our simulation in case of NC-InPacket and HARQ full ReTx in LTE under the assumption that all retransmission are used. At the initial transmission, both schemes transmit the same packet, but instead of a full packet as retransmission, like in HARQ full ReTx, NC-InPacket transmit a size reduced packet. For that, the packet $\mathbf{c}_{i}^{r}$ generated by the common LTE encoder is split into two parts $\mathbf{c}_{i, 1}^{r}$ and $\mathbf{c}_{i, 2}^{r}$. These parts are combined by a bitwise XOR operation given by

$$
\mathbf{c}_{i, \mathrm{NC}}^{r}=\mathbf{c}_{i, 1}^{r} \oplus \mathbf{c}_{i, 2}^{r},
$$

to exploit the whole information given by a full packet. Due to the combination of two parts of $\mathbf{c}_{i}^{r}$, the size of the retransmission packet is reduced by 2 . This procedure can be interpreted as generating a product code, if the parts are valid codewords. The turbo encoder performs the horizontal encoding of every part $\mathbf{c}_{i, 1}^{r}$ and $\mathbf{c}_{i, 2}^{r}$ and the retransmission is a vertical $2 / 3$ single parity check (SPC) code. The transmission procedure, is given as follows, where $\nu$ is the transmission index, i.e., $\nu=0$ for the initial transmission and higher $\nu$ for retransmissions. 
1) The codeword $\mathbf{c}_{i}^{r}$ with $\nu=0$ is transmitted in time slot $t_{i}$ and $\mathbf{y}_{i}$ as in (2) is received and decoded. In case of a decoding failure, the eNB sends $\mathrm{NAK}_{i}$ to the UE. If decoding is successful an $\mathrm{ACK}_{i}$ is transmitted and the procedure starts with a new packet $\mathbf{c}_{i+1}^{r}$.

2) If NAK. For the first retransmission $\nu=1$, a $\mathrm{NC}$ retransmission $\mathbf{c}_{i, \mathrm{NC}}^{r}$ generated by (4) is transmitted in time slot $t_{j}$ by the UE and $\mathbf{y}_{j}$ is received at the eNB.

3) From both received signals, $\mathbf{y}_{i}$ and $\mathbf{y}_{j}$, the eNB tries to reconstruct the codeword $\mathbf{b}_{i}$ as it will be described in Section IV.

4) Depending on the decoding success, the eNB transmits an ACK/NAK to the UE.

5) If NAK, then in the next two retransmissions only one part $\mathbf{c}_{i, 1}^{r}$ or $\mathbf{c}_{i, 2}^{r}$, depending on $\nu$, is transmitted, shown in Fig 2.

6) If NAK, after two retransmissions, this procedure starts again with point 2)

\section{B. $H A R Q$ with half retransmission size (HARQ half ReTx)}

As a benchmark, a simple retransmission scheme called HARQ half ReTx is proposed instead of using LTE own reducing techniques. In the LTE uplink, the adaptive reduction of retransmission within one HARQ process is not provided, but the LTE downlink offers a reduction by pruning the according redundancy version of the transmission. This pruning is done in the Rate Matching block in Fig. 1 by selecting less bits than the initial transmission. Eq. (5) shows in principle the punctering vector used by LTE rate matching.

$$
\begin{aligned}
& P_{\mathrm{LTE}}(\nu=0)=\left[\begin{array}{lllllllll}
1 & 1 & 1 & 1 & \ldots & 1 & 1 & 1 & 1
\end{array}\right] \\
& P_{\mathrm{LTE}}(\nu \geq 0)=\left[\begin{array}{llllllll}
1 & 1 & 1 & 1 & \ldots & 0 & 0 & 0
\end{array}\right]
\end{aligned}
$$

$P$ is the puncturing vector. Especially for Chase Combining the reduced version selected by this LTE scheme is always the first part of the initial transmission. The behavior of selecting only the first part is avoided by introducing an additional puncturing on top of a full size retransmission shown in Eq. (6).

$$
\begin{aligned}
& P_{\text {half }}(\nu=1)=\left[\begin{array}{lllllllll}
1 & 0 & 1 & 0 & 1 & 0 & 1 & 0
\end{array}\right] \\
& P_{\text {half }}(\nu=2)=\left[\begin{array}{lllllllll}
0 & 1 & 0 & 1 & 0 & 1 & 0 & 1
\end{array}\right]
\end{aligned}
$$

At the initial transmission with $\nu=0$ all bits generated by the common LTE encoder will be transmitted $\left(P_{\text {half }}=P_{\mathrm{LTE}}(\nu=\right.$ $0)$ ). For higher transmission indices $\nu>0$ we skip every second bit depending on the retransmission number shown in eq. (6). After $\nu=2$ retransmissions the same bits are transmitted as in one common LTE retransmission, due to the disjunct structure of the puncturing masks.

\section{Impact of system parameters}

As mentioned above, LTE defines segmentation by splitting the information word $\mathbf{a}_{i}$ into $N_{\mathrm{s}}$ segments $\mathbf{a}_{i, s}$. Due to the separate encoding, the decoding of each segment is done also separately. If we further split the retransmission packet into parts as introduced in Section III we can use these segments as part $\mathbf{c}_{i, 1}^{r}$ and $\mathbf{c}_{i, 2}^{r}$, if we restricted the transport formats (TFs) with $N_{\mathrm{s}}=2$. The decoding could be done very easily, because every part is a valid transmission codeword generated by the common LTE turbo encoder. In the sequel, we assume that every packet $\mathbf{c}_{i}^{r}$ contains $N_{\mathrm{s}}=2$ segments.

LTE introduces cyclic redundancy check (CRC) code for every segment $\mathbf{c}_{i, s}^{r}$ and for the whole packet $\mathbf{c}_{i}^{r}$ to check a decoding success. The acknowledgment ACK/NAK is transmitted, if any segment or the CRC for the whole packet gives an error. Note that, the ACK/NAK do not signal which segment is erroneous.

\section{Network Coded HARQ over different packets}

We proposed a comparable scheme to NC-InPacket, called NC-HARQ in [8] and [9]. In that scheme, two packets out of different HARQ processes of one user are combined into one retransmission packet by $\mathrm{NC}$. The important difference of both NC approaches is the signaling of erroneous packets. NC-HARQ provides ACK/NAK signaling for every packet. However, as the signaling of decoding success of segments is not defined in case of NC-InPacket, it is not possible to signal the UE the decoding success of one special segment for its own.

\section{Decoder Algorithm}

\section{A. NC-InPacket Decoder}

The decoder structure of NC-InPacket is illustrated in Fig. 3 for two parts. Note, as LTE introduces segmentation, the introduced parts could be implemented easily by using directly segments coming from the LTE encoder. In the sequel, we explain the procedure for two segments, but it can be generalized to use more segments. Based on the received signals $\mathbf{y}_{i}$ and $\mathbf{y}_{j}$ we calculate the LLRs $\mathrm{L}_{\mathrm{Dem}}\left(\mathbf{c}_{i, 1}^{r}\right)$ and $\mathrm{L}_{\operatorname{Dem}}\left(\mathbf{c}_{i, 2}^{r}\right)$ for the initial transmission and $\mathrm{L}_{\mathrm{Dem}}\left(\mathbf{c}_{i, \mathrm{NC}}^{r}\right)$ for the retransmission by using (3). The rate dematching block maps the LLRs of the codebits to the LLRs of the mother code by deinterleaving and depuncturing.

The decoding steps are very similar as described in [9]. Hence, the procedure is only introduced very briefly here. Assuming the first transmission is erroneous, then the procedure is done as follows:

1) Decoding $\mathbf{b}_{i, 1}$ and $\mathbf{b}_{i, 2}$ : After $N_{\mathrm{H}}$ iterations the extrinsic information $\mathrm{L}_{\mathrm{Ext}}\left(\mathbf{a}_{i, 1}\right)$ and $\mathrm{L}_{\mathrm{Ext}}\left(\mathbf{a}_{i, 2}\right)$ is obtained at the output of the turbo decoders $\mathcal{C}_{1}^{-1}$ and $\mathcal{C}_{2}^{-1}$.

2) Due to the linearity of all used operations, like rate matching, XOR combination and LTE turbo encoding, the retransmitted packet $\mathbf{c}_{i, \mathrm{NC}}^{r}$ is also a valid LTE retransmission. Hence, the combination of the extrinsic information $\mathrm{L}_{\mathrm{Ext}}\left(\mathbf{a}_{i, 1}\right)$ and $\mathrm{L}_{\mathrm{Ext}}\left(\mathbf{a}_{i, 2}\right)$ yields a-priori information for the $\mathrm{NC}$ retransmission. The combination of LLRs is done by the boxplus operation [10]. Therefore, we calculate a-priori information and use it at the decoder of the retransmitted packet by

$$
\mathrm{L}_{\mathrm{a}}\left(\mathbf{a}_{i, \mathrm{NC}}\right)=\mathrm{L}_{\mathrm{Ext}}\left(\mathbf{a}_{i, 1}\right) \boxplus \mathrm{L}_{\mathrm{Ext}}\left(\mathbf{a}_{i, 2}\right) .
$$


3) The turbo decoder $\mathcal{C}_{\mathrm{NC}}^{-1}$ of the retransmission codeword $\mathbf{c}_{i, \mathrm{NC}}^{r}$ provides also a-priori information for the other decoders, given by

$$
\begin{aligned}
& \mathrm{L}_{\mathrm{a}}\left(\mathbf{a}_{i, 1}\right)=\mathrm{L}_{\mathrm{Ext}}\left(\mathbf{a}_{i, 2}\right) \boxplus \mathrm{L}_{\mathrm{Ext}}\left(\mathbf{a}_{i, \mathrm{NC}}\right) \\
& \mathrm{L}_{\mathrm{a}}\left(\mathbf{a}_{i, 2}\right)=\mathrm{L}_{\mathrm{Ext}}\left(\mathbf{a}_{i, 1}\right) \boxplus \mathrm{L}_{\mathrm{Ext}}\left(\mathbf{a}_{i, \mathrm{NC}}\right)
\end{aligned}
$$

4) The overall procedure is done in an iterative way, $N_{\mathrm{V}}$ denotes the iteration number of the vertical SPC code. At the end, we obtain the LLRs $\operatorname{L}_{\operatorname{Dec}}\left(\mathbf{a}_{i, 1}\right)$ and $\operatorname{L}_{\operatorname{Dec}}\left(\mathbf{a}_{i, 2}\right)$ which are concatenated, to one final LLR $\operatorname{LDec}_{\operatorname{Dec}}\left(\mathbf{a}_{i}\right)$ as estimation $\hat{\mathbf{a}}_{i}$.

\section{B. HARQ half ReTx Decoder}

The HARQ half ReTx decoder is the common LTE turbo decoder with the difference of the depuncturing before decoding. For that, only low additional complexity is added.

\section{Performance Analysis}

In this section, we present the simulation results in an LTE link-level uplink simulator chain regarding the different retransmission schemes. The detailed simulation parameters setup of the LTE uplink simulator is summarized in Table I and Table II shows the used transport formats (TFs) in this paper.

TABLE I

SYSTEM PARAMETERS FOR LTE LINK-LEVEL UPLINK SIMULATION CHAIN

\begin{tabular}{|c|c|}
\hline parameters & setup \\
\hline Carrier frequency & $2.3 \mathrm{GHz}$ \\
Modulation & $16-\mathrm{QAM}$ \\
Turbo code rate & $1 / 3$ \\
Number of transmit antennas UE & 1 \\
Number of receive antennas eNB & 2 \\
Channel & AWGN \\
& Rayleigh fading, UE speed = 50kmh \\
Max. number of retransmissions & HARQ full ReTx: 4, \\
& HARQ half ReTx: 8, \\
& NC-InPacket: 8, NC-HARQ: 8 \\
\hline
\end{tabular}

For the schemes with half retransmission size the maximum number of retransmissions is doubled, due to the same number of transmitted bits, also illustrated in Fig. 2. The performance of CC and IR differs only slightly in case of low code rates [11]. IR introduces coding gain by adding redundancy, whereas $\mathrm{CC}$ introduces SNR gain. Therefore, we focus on TFs with high effective code rates $R_{\mathrm{C}}$.

TABLE II

ADDITIONAL SYSTEM PARAMETER FOR DIFFERENT SIMULATIONS

\begin{tabular}{|c|c|c|c|c|}
\hline Transport Format & $N_{\mathrm{a}}$ & $N_{\mathrm{C}}$ & eff. code rate $R_{\mathrm{C}}$ & $N_{\mathrm{S}}$ \\
\hline 104 & 2152 & 2640 & 0.82 & 1 \\
\hline 356 & 11448 & 14256 & 0.80 & 2 \\
\hline
\end{tabular}

As main performance measurement the normalized throughput is considered, given by

$$
\eta=\frac{N_{\text {correct }}}{N_{\text {full,length }}+0.5 N_{\text {half,length }}},
$$

where $N_{\text {correct }}$ indicates the number of correctly decoded blocks, whereas $N_{\text {full,length }}$ and $N_{\text {half,length }}$ denote the number of blocks transmitted with length $N_{\mathrm{c}}$ and the half length $0.5 N_{\mathrm{c}}$.

\section{A. HARQ half ReTx as benchmark}

HARQ half ReTx introduces intermediate throughput levels due to the smaller retransmissions sizes, e.g. if a packet is erroneous in the first transmission and the system needs one additional retransmission with full length in a HARQ full ReTx system, the normalized throughput is $\eta\left(\nu_{\mathrm{f}}=1\right)=\frac{1}{2}$. However, if a retransmitted packet with half of the length yields already a decoding success, the throughput becomes $\eta\left(\nu_{\mathrm{h}}=1\right)=\frac{1}{1+0.5}=\frac{2}{3}$, with $\nu_{\mathrm{f}}$ and $\nu_{\mathrm{h}}$ as retransmission index for HARQ full ReTx or HARQ half ReTx, respectively. Fig. 4 shows the normalized throughput of HARQ half ReTx with $N_{\mathrm{s}}=2$ and HARQ full ReTx with TF 356 for CC and IR. Additionally, the performance of the LTE reduced ReTx is shown. This figure also shows the discrete throughput levels $\eta\left(\nu_{\text {scheme }}\right)$ for each scheme on the right ordinate.

As expected, the simple puncturing scheme from Sec. III outperforms the LTE reducing ReTx scheme. In the CC case the gain starts at the $\nu=2$ retransmission, due to the altern structure of the puncturing matrix in (6). In the IR case, the main difference is starting with retransmission $\nu=4$ due to the limited definition of 4 different RV in LTE [1].

In the sequel, we only focus on the the simple puncturing. Here, the normalized throughput of HARQ half ReTx behaves very similar to the HARQ full ReTx scheme, but introduces intermediate levels. Due to this levels HARQ half ReTx outperforms HARQ full ReTx, by terms of throughput performance. Regarding only one retransmissions $\nu=1$, the throughput performance gain of HARQ half ReTx compared to HARQ full ReTx is 33\% for a SNR region of approximately $1.5 \mathrm{~dB}$ for $\mathrm{CC}$ and more than $3.5 \mathrm{~dB}$ for IR. However, HARQ half ReTx performs in some SNR regions similar to HARQ full $\operatorname{ReTx}$, due to the same size of overall retransmissions. But it always performs better or equal than HARQ full ReTx. For that, we only compare the results with respect to the HARQ half ReTx scheme in the sequel. It can also observed that in both schemes IR outperforms CC.

\section{B. NC-InPacket}

Fig. 5 shows the normalized throughput of NC-InPacket together with the results for HARQ half ReTx of Fig. 4 in an AWGN scenario. At a specific SNR region, denoted with NC-InPacket gain region $\mathrm{CC}$, it can be observed that NCInPacket CC outperforms HARQ half ReTx CC. For this SNR, $\mathrm{NC}$-InPacket $\mathrm{CC}$ can recover one packet by only one reduced size retransmission, while HARQ half ReTx CC requires more retransmissions. At an SNR $<4.5 \mathrm{~dB}$ HARQ half ReTx always outperforms NC-InPacket CC. In contrast, NC-InPacket IR never outperforms HARQ half ReTx IR. It can be observed that the main loss starts with the second retransmission, i.e. at $\nu>2$. This loss of NC-InPacket compared to HARQ half ReTx is due to the missing signaling of decoding success of each segment in LTE. If one segment is decoded successfully 


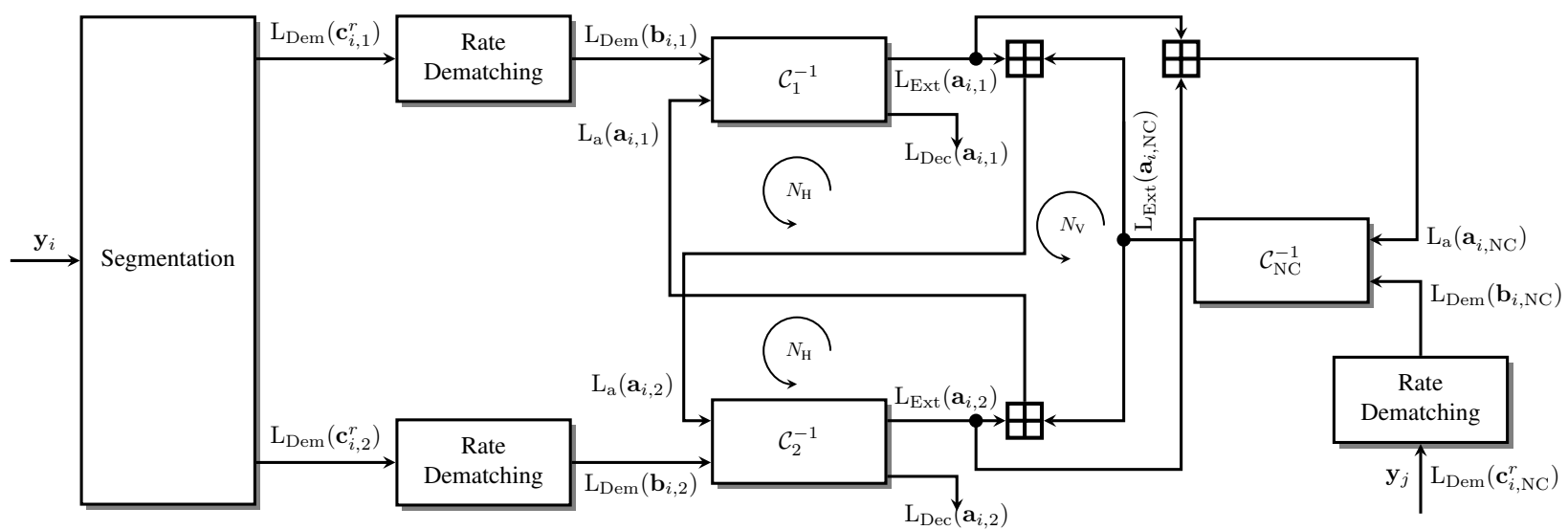

Fig. 3. Generalized iterative decoding structure for NC-InPacket, where $\boxplus$ denotes the boxplus operation.

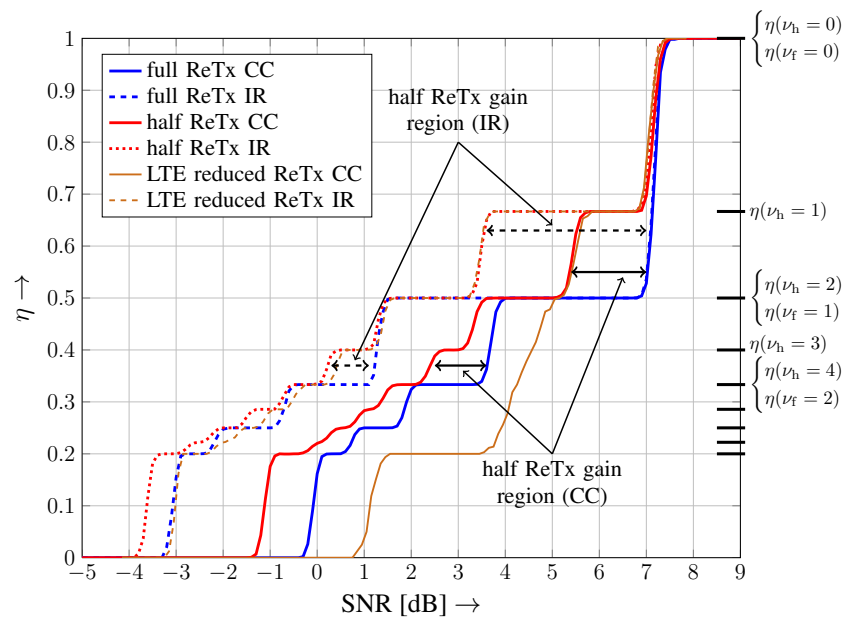

Fig. 4. Throughput performance of HARQ full ReTx vs. HARQ half ReTx with TF 356 with an AWGN channel. Additionally the performance of the LTE reducing technique is shown. On the right ordinate, the discrete throughput levels are shown. $\nu_{\mathrm{h}}$ for HARQ half ReTx and $\nu_{\mathrm{f}}$ for HARQ full ReTx.

but another segment is in error, the receiver transmit a NAK for the whole packet, and a whole packet will be retransmitted, this reduces the throughput performance.

In Fig. 6 we compare the IR schemes in a Rayleigh fading channel scenario. Note, as described in the AWGN scenario the main loss starts with the second retransmission. In a Rayleigh fading scenario the channel influences the transmission scheme in such a way that retransmissions occur also in a higher SNR region. For that, HARQ half ReTx IR always outperforms NCInPacket.

\section{NC-HARQ}

Fig. 7 shows the throughput performance of NC-HARQ, also presented in [8], and HARQ half ReTx with TF 104 in an AWGN scenario. Here, NC-HARQ outperforms HARQ half ReTx for both retransmission philosophies, CC and IR. In contrast to NC-InPacket, NC-HARQ is able to signal a decoding success for every part of a combined retransmission. For that, NC-HARQ outperforms HARQ half ReTx with a retransmission index higher than $\nu>2$. In the area with one to two retransmissions $1 \leq \nu \leq 2$ HARQ half ReTx

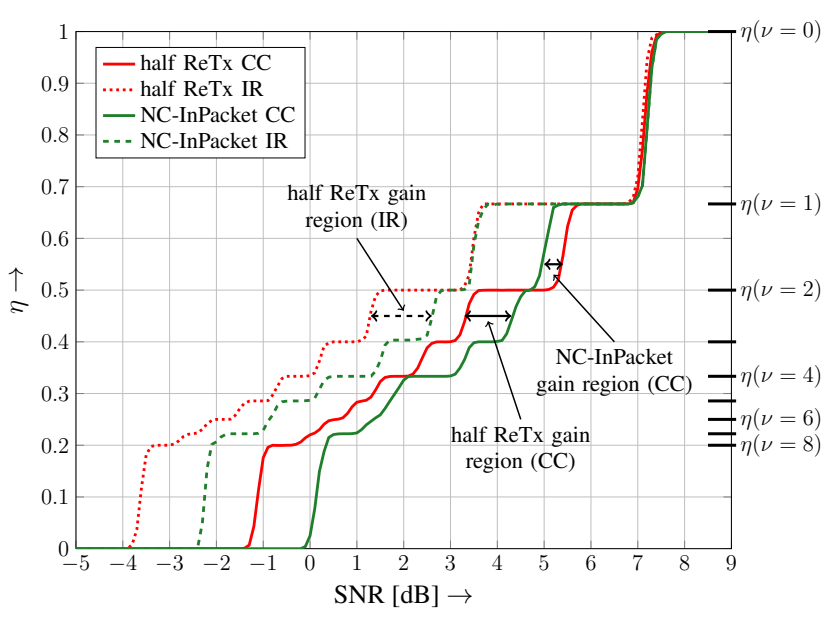

Fig. 5. Throughput performance of NC-InPacket vs. HARQ half ReTx with TF356 with an AWGN channel. The right ordinate shows the possible discrete throughput levels. HARQ half ReTx and In-Packet have the same levels.

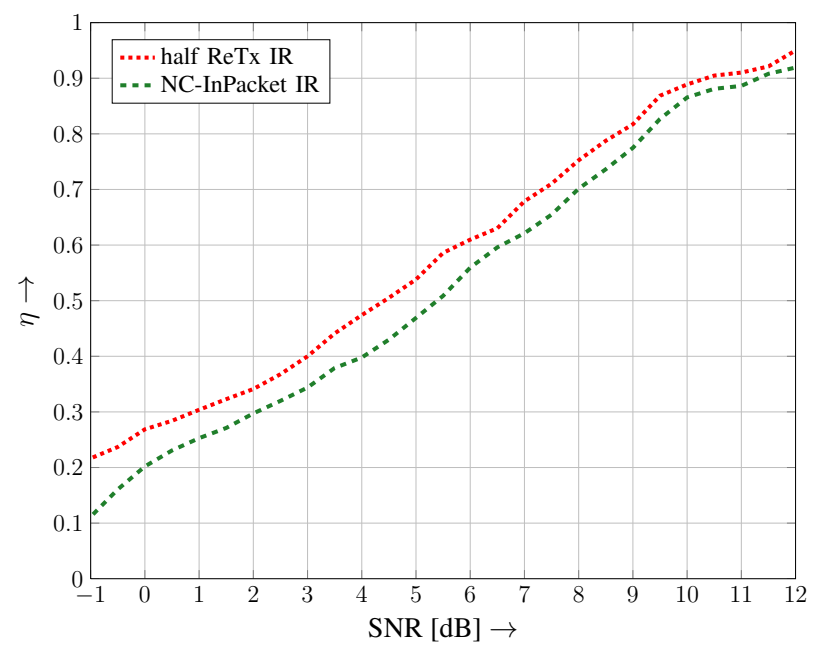

Fig. 6. Throughput performance of NC-InPacket vs. HARQ half ReTx with TF 356 with a 1-path Rayleigh fading channel with a UE speed of $50 \mathrm{kmh}$.

IR is the upper limit of NC-HARQ IR and CC, this result is similar to the NC-InPacket scheme in Fig. 5. Note that, these two systems are designed identical for the initial transmission 
and the first retransmission. Mainly, both systems differ in the signaling of ACK/NAK and timing of the transmitted packets. The main difference arise with the second retransmission. There, NC-HARQ gains due to the possible combination of different erroneous packets, i.e., the NC combination of two packets is done in a flexible way, meaning, if one packet is correct, the retransmission of the other packet is done with a new packet. In contrast, NC-InPacket is a fixed scheme, i.e., due to the missing signaling of the decoding success of one segment, the overall packet has to be correct to transmit new information.

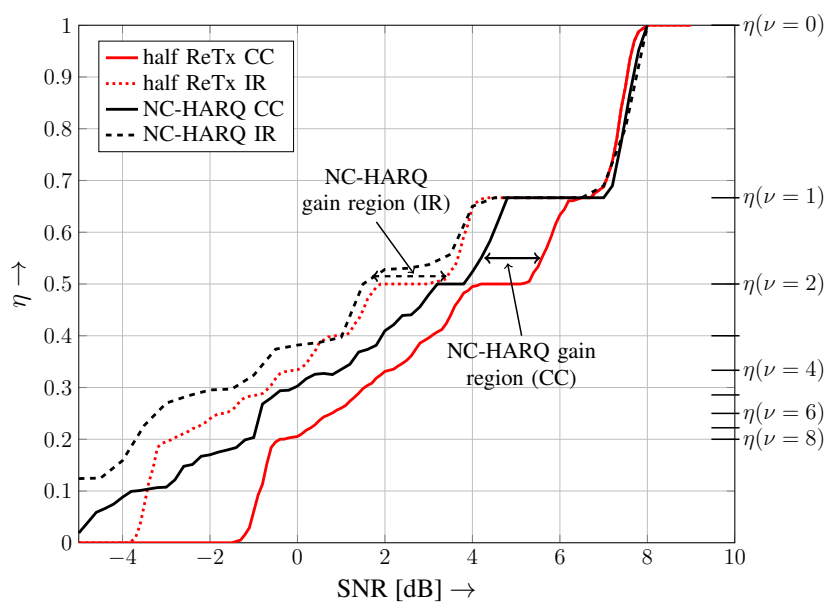

Fig. 7. Throughput performance of NC-HARQ vs. HARQ half ReTx with TF 104 with a AWGN channel. The right ordinate shows the possible discrete throughput levels.

Similar to Fig. 6 the results of a Rayleigh fading channel of NC-HARQ IR and HARQ half ReTx IR are shown in Fig. 8. For higher SNR region, HARQ half ReTx IR outperforms the NC-HARQ scheme, similar to the NC-InPacket scheme, but for a lower SNR region the NC-HARQ scheme outperforms HARQ half ReTx IR, due to the flexible combination of retransmission packets.

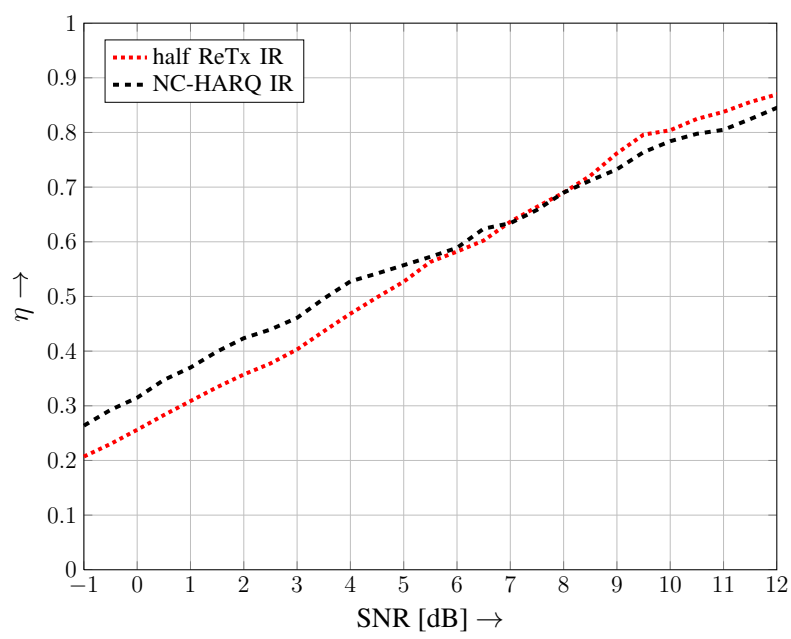

Fig. 8. Throughput performance of NC-HARQ vs. HARQ half ReTx with TF 104 a 1-path Rayleigh fading channel with a UE speed of $50 \mathrm{kmh}$.

\section{CONCLUSION}

In this paper we have considered three different retransmission schemes in comparison to HARQ with full size ReTx in LTE. In HARQ full ReTx, the retransmission of an erroneous detected packet has the same size than the initial transmission. NC-InPacket uses network coding to generate a retransmission packet, whereas HARQ half ReTx implements an additional puncturing on top of the LTE packet. Therefore, both schemes reduce the size of the retransmission packet. In contrast to the NC-HARQ scheme proposed in [8], which combines two packets out of different HARQ processes, NC-InPacket only outperforms HARQ half ReTx in a very small SNR region in terms of throughput performance for Chase Combining with a gain up to $33 \%$. In the other SNR regions no throughput gain is obtained, due to the limitations of the ACK/NAK signaling in LTE. For that the puncturing scheme HARQ half ReTx which only introduces a vanishing additional complexity have an overall better performance. Additionally, we have compared the simple HARQ half ReTx scheme with the previous published NC-HARQ system. The main improvement of NC-HARQ in comparison to HARQ half ReTx is obtained with the second retransmission, due to the flexible combination of different packets. If the LTE standard would implement segment-based ACK/NAKs, than a similar behavior of NCInPacket to NC-HARQ would be possible.

\section{REFERENCES}

[1] 3GPP TS 36.212 V9.2.0, "Multiplexing and Channel Coding," Jun. 2010.

[2] R. Ahlswede, N. Cai, S.-Y. R. Li, and R. W. Yeung, "Network Information Flow," IEEE Trans. on Information Theory, , no. 4, Jul. 2000.

[3] P. Larsson, N. Johansson, and K.-E. Sunell, "Coded Bi-directional Relaying," in IEEE Vehicular Technology Conference (VTC 2006Spring), Melbourne, Australia, May 2006.

[4] P. Larsson and N. Johansson, "Multi-User ARQ," in IEEE Vehicular Technology Conference (VTC 2006-Spring), Melbourne, Australia, May 2006.

[5] P. Larsson, "Multicast Multiuser ARQ," in IEEE Wireless Communications and Networking Conference (WCNC08), Las Vegas, USA, Apr. 2008.

[6] M. Ghaderi, D. Towsley, and J. Kurose, "Network Coding Performance for Reliable Multicast," in IEEE Military Communications Conference (MILCOM07), Florida, USA, Oct. 2007.

[7] D. Nguyen, T. Tran, T. Nguyen, and B. Bose, "Wireless Broadcast Using Network Coding," IEEE Transactions on Vehicular Technology, vol. 58 , no. 2, pp. 914-925, Feb. 2009

[8] Y. Lang, D. Wübben, A. Dekorsy, V. Braun, and U. Doetsch, "Improved HARQ based on Network Coding and its Application in LTE," in IEEE Wireless Communications and Networking Conference (WCNC 2012), Paris, France, Apr. 2012.

[9] Y. Lang, D. Wübben, A. Dekorsy, V. Braun, and U. Doetsch, "A TurboLike Iterative Decoding Algorithm for Network Coded HARQ," in 9th International ITG Conference on Source and Channel Coding (SCC13), Munich, Germany, Jun. 2013.

[10] J. Hagenauer, "Soft is better than Hard," Communications, Coding and Cryprology, 1994.

[11] Jung-Fu Cheng, "On the coding gain of incremental redundancy over chase combining," in IEEE Global Telecommunications Conference, 2003. (GLOBECOM03), Dec. 2003. 